\title{
The effects of enteral and parenteral nutrition on clinical outcomes in cancer inpatients receiving palliative care
}

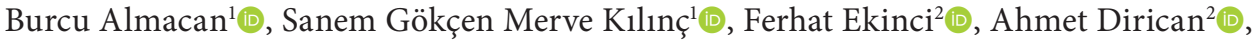 \\ Gamze Göksel Öztürk² ${ }^{2}$, Atike Pınar Erdoğan²® \\ ${ }^{1}$ Department of Internal Medicine, Medicine Faculty of Celal Bayar University, Manisa, Turkey \\ ${ }^{2}$ Department of Internal Medicine, Division of Oncology, Medicine Faculty of Celal Bayar University, Manisa, Turkey
}

\begin{abstract}
Objectives: This study aims to determine the effects of enteral (EN) and parenteral nutrition (PN) on clinical outcomes in cancer inpatients receiving palliative care in the oncology unit.

Patients and methods: A total of 100 cancer patients who were admitted to the Manisa Celal Bayar University Oncology Inpatient Unit between January 2016 and December 2017 for palliative support were included in our study.

Results: There was no difference between EN and PN in terms of general mortality. According to patient characteristics, the rates of receiving chemotherapy and radiotherapy were equivalent. Parenteral nutrition may cause increased rate of central line infection due to growth in blood and urine culture. It may also disrupt fluid electrolyte balance.

Conclusion: Patients should be closely monitored for complications that may arise during parenteral and enteral nutritional therapy. Keywords: Enteral nutrition, palliative care, parenteral nutrition.
\end{abstract}

Sufficient nutrition and positive caloric balance are among the most important factors determining quality of life and life expectancy in cancer patients. ${ }^{[1]}$ About $20-40 \%$ of cancer patients die due to severe malnutrition during the course of their disease. Negative nitrogen balance in cancer patients should be corrected and replaced by providing adequate calorie, protein, vitamin and mineral support. ${ }^{[2]}$ In cancer patients whose nutritional deficiencies cannot be corrected, enteral or parenteral nutritional supplement products can be used. ${ }^{[3]}$ Therefore, we planned this study to determine the effects of enteral (EN) and parenteral nutrition (PN) on clinical outcomes in cancer inpatients receiving palliative care in the oncology unit.

\section{PATIENTS AND METHODS}

This study included 100 cancer patients who were admitted to the Manisa Celal Bayar University Oncology Inpatient Unit between January 2016 and December 2017 for palliative support. Age, sex, nicotine use, additional diseases, blood tests at admission, and history of chemotherapy and radiotherapy of the patients were noted. Enteral or parenteral method of feeding, length of hospital stay, presence of growth in cultures, and history of embolization were investigated and noted. All statistical data were analyzed with SPSS version 15.0 software (SPSS Inc., Chicago, IL, USA). $\mathrm{P}<0.05$ was considered level of statistical significance. Student's t-test was used

Received: June 09, 2020 Accepted: July 08, 2020 Published online: August 25, 2020

Correspondence: Burcu Almacan, MD. Celal Bayar Üniversitesi Tıp Fakültesi İç Hastalıkları Anabilim Dalı, 45030 Manisa, Türkiye. Tel: +90 554 - 5530778 e-mail: Burcu_almazcon@hotmail.com 
to assess continuous variables, while chi-square was used for categorical variables.

\section{RESULTS}

According to the demographic data of the 100 patients included in the study, the number of patients receiving PN was significantly higher among male patients compared to female patients (Table 1). Mean patient age was 57.28 (36-82) years in patients receiving $\mathrm{EN}$, and $60.78(30-82)$ among patients receiving $\mathrm{PN}$. There was no significant difference between EN and PN groups according to general mortality. When patient characteristics were evaluated, the chemotherapy and radiotherapy rates were equivalent. There was no difference between the groups according to additional diseases (Table 1). It was $27 \%$ in the PN group and $8.3 \%$ in the EN group. The number of patients requiring intensive care was significantly higher in the PN group compared to the EN group $(p=0.036)$. Thrombosis rates were similar among both groups. Central catheter infection complication in PN group compared to EN group; it was statistically significantly higher $(p=0.002)$. Blood culture infection complication in PN group compared to EN group; it was statistically significantly higher $(p=0.002)$. Urine culture infection complication in PN group compared to EN group; it was statistically significantly higher $(p=0.001)$. There was no significant difference between the groups according to length of hospital stay. Laboratory results at admission and 48 hours after hospitalization were compared using Wilcoxon Signed Ranks test. Creatinine and $\mathrm{Na}$ values were significantly higher 48 hours after hospitalization compared to admission values. Albumin levels were significantly lower at 48 hours compared to admission $(p=0.08)$ (Table 2).

\section{DISCUSSION}

In this study, we investigated the general effects of nutritional support on clinical outcomes in cancer patients. Cachexia is a multifactorial syndrome characterized by severe body weight, muscle and fat loss, due to increased protein catabolism caused by the underlying disease. Cachexia is a serious condition as it causes increased morbidity and mortality. ${ }^{[3]}$ Adequate metabolic and nutritional support should be provided to prevent cachexia in cancer patients. In cancer patients whose malnutrition cannot be corrected, enteral or parenteral nutrition supplement products can be used. Nutritional support should be initiated in suitable patients by evaluating the potential risks and complications. We did not encounter a difference between enteral and parenteral nutrition in terms of mortality in cancer inpatients receiving palliative care in the oncology unit. In a meta-analysis by Elke et al., ${ }^{[4]}$ no significant difference was observed between the

Table 1. Age, sex, comorbidity, history of chemotherapy or radiotherapy, length of stay, mortality, and thrombosis history of the patients

\begin{tabular}{|c|c|c|c|c|c|c|}
\hline & \multicolumn{3}{|c|}{ Parenteral nutrition } & \multicolumn{3}{|c|}{ Enteral nutrition } \\
\hline & $\mathrm{n}$ & $\%$ & Mean \pm SD & $\mathrm{n}$ & $\%$ & Mean \pm SD \\
\hline Age (year) & & & $59.0 \pm 10.7$ & & & $60.0 \pm 12.7$ \\
\hline \multicolumn{7}{|l|}{ Sex } \\
\hline Female & 21 & 33.3 & & 20 & 55.6 & \\
\hline Male & 42 & 66.7 & & 16 & 44.4 & \\
\hline Chemotherapy & 53 & 62.4 & & 32 & 37.6 & \\
\hline Radiotherapy & 18 & 29 & & 11 & 30.6 & \\
\hline Mortality & 13 & 21 & & 3 & 8.3 & \\
\hline Thrombosis & 3 & 4.8 & & 1 & 2.8 & \\
\hline Diabetes mellitus & 13 & 20.6 & & 9 & 25.0 & \\
\hline Hypertension & 16 & 25.4 & & 7 & 19.4 & \\
\hline Chronic kidney disease & 9 & 14.3 & & 8 & 22.2 & \\
\hline Coronary artery disease & 7 & 11.1 & & 5 & 13.9 & \\
\hline Length of hospital stay & & & $7.0 \pm 7.6$ & & & $7.0 \pm 13.3$ \\
\hline
\end{tabular}


Table 2. Laboratory values at admission and 48 hours after admission

\begin{tabular}{lcccc}
\hline & Admission laboratory results & & 48-hour laboratory results & \\
\cline { 2 - 2 } Neutrophil & Mean \pm SD & & Mean \pm SD & $p$ \\
\hline Lymphocyte & $5011.18 \pm 2880.764$ & & $5425.73 \pm 3150.413$ & 0.846 \\
C-reactive protein & $1423.38 \pm 1018.877$ & & $1520.27 \pm 987.353$ & 0.477 \\
Albumin & $6.90 \pm 9.930$ & & $8.52 \pm 10.193$ & 0.789 \\
Alanine aminotransferase & $3.3267 \pm 0.73560$ & & $3.09 \pm 0.762$ & 0.08 \\
Creatinine & $24.85 \pm 23.904$ & & $22.03 \pm 20.002$ & 0.361 \\
Sodium & $0.887 \pm 0.4024$ & & $1.08 \pm 0.490$ & 0.049 \\
Potassium & $136.33 \pm 3.789$ & & $137.61 \pm 2.825$ & 0.018 \\
International normalized ratio & $4.15 \pm 0.651$ & & $4.03 \pm 0.706$ & 0.668 \\
\hline
\end{tabular}

two nutrition groups according to mortality. ${ }^{[5-7]}$ Enteral nutrition contributes to the maintenance of biological, immunological and chemical barrier balance of intestinal mucosa cells. Jilaling Shi et al. ${ }^{[6]}$ reported that hospital-acquired respiratory tract infections significantly decreased in patients receiving enteral nutrition due to the activation of lymphoid tissue found in the gastrointestinal system. ${ }^{[8]}$ In our study, according to infectious complications were significantly higher in terms of central line, blood culture, and urine culture growth among patients receiving PN compared to those receiving EN. Elke et al., ${ }^{[4]}$ found an increase in infectious parameters. ${ }^{[7]}$ In a metaanalysis of 37 studies involving patients receiving long-term parenteral nutrition, the most common complication was catheter infection and catheter sepsis. ${ }^{[7]}$ However, there are studies showing that the incidence of infectious complications in critical patients may be reduced by contemporary care in intensive care units. ${ }^{[8-10]}$ Enteral nutrition is associated with a potentially low nutritional capability in end-stage terminal cancer patients with impaired oral intake and gastrointestinal dysfunction. Studies show that enterally fed patients receive less than $60 \%$ of the average prescribed calories. ${ }^{[11]}$ Parenteral nutrition is the primary therapeutic nutritional support to prevent metabolic degradation and loss of body mass in these critical patients. ${ }^{[4]}$ Studies have shown that patients receiving EN were fed with a lower calorie and nutritional content compared to $\mathrm{PN} .{ }^{[8]}$ Shi et al. ${ }^{[6]}$ reported that $\mathrm{PN}$ provided rapid clinical improvement in critical patients, reducing the length of hospital stay. In our study, we did not observe a significant difference between the two groups according to length of hospital stay. Albumin was used to evaluate the patient's nutritional status. However, there was no significant difference between admission and post-admission albumin values in either group. In our study, the need for intensive care was significantly higher in the PN group compared to the EN group. Fluid and electrolyte balance may be disrupted during parenteral feeding. ${ }^{[7]}$ We regularly monitored our patients for fluid, electrolyte imbalance, high blood sugar levels, and cardiovascular dysfunction that may occur during parenteral nutrition. We also found that the creatinine and $\mathrm{Na}$ values of the patients were significantly higher at post-admission compared to admission values. Hypophosphatemia, hypokalemia, hypomagnesemia and arrhythmia were not observed. We did not observe a difference in terms of blood sugar. Refeeding syndrome, characterized by biochemical and metabolic abnormalities following nutrition, was not observed in any of our patients. There was no significant difference in admission and postadmission liver enzymes and bilirubin levels in either group.

In conclusion parenteral nutrition may lead to increased blood culture and urine culture growth and central line infections and may disrupt fluid electrolyte balance. Patients should be closely monitored for complications that may arise during treatment.

\section{Declaration of conflicting interests}

The authors declared no conflicts of interest with respect to the authorship and/or publication of this article. 


\section{Funding}

The authors received no financial support for the research and/or authorship of this article.

\section{REFERENCES}

1. Planas M, Álvarez-Hernández J, León-Sanz M, CelayaPérez S, Araujo K, García de Lorenzo A; PREDyCES ${ }^{\circledR}$ researchers. Prevalence of hospital malnutrition in cancer patients: a sub-analysis of the PREDyCES ${ }^{\circledR}$ study. Support Care Cancer 2016;24:429-35.

2. Van Lancker A, Velghe A, Van Hecke A, Verbrugghe M, Van Den Noortgate N, Grypdonck $\mathrm{M}$, et al. Prevalence of symptoms in older cancer patients receiving palliative care: a systematic review and meta-analysis. J Pain Symptom Manage 2014;47:90-104.

3. Muscaritoli M, Molfino A, Gioia G, Laviano A, Rossi Fanelli F. The "parallel pathway": a novel nutritional and metabolic approach to cancer patients. Intern Emerg Med 2011;6:105-12.

4. Elke G, van Zanten AR, Lemieux M, McCall M, Jeejeebhoy KN, Kott M, et al. Enteral versus parenteral nutrition in critically ill patients: an updated systematic review and meta-analysis of randomized controlled trials. Crit Care 2016;20:117.

5. Lewis SR, Schofield-Robinson OJ, Alderson P, Smith AF. Enteral versus parenteral nutrition and enteral versus a combination of enteral and parenteral nutrition for adults in the intensive care unit. Cochrane
Database Syst Rev 2018;6:CD012276.

6. Shi J, Wei L, Huang R, Liao L. Effect of combined parenteral and enteral nutrition versus enteral nutrition alone for critically ill patients: A systematic review and meta-analysis. Medicine (Baltimore) 2018;97:e11874.

7. Hartl WH, Jauch KW, Parhofer K, Rittler P. Complications and monitoring - Guidelines on Parenteral Nutrition, Chapter 11. Ger Med Sci 2009;7:Doc17.

8. Gavri C, Kokkoris S, Vasileiadis I, Oeconomopoulou AC, Kotanidou A, Nanas S, et al. Route of nutrition and risk of blood stream infections in critically ill patients; a comparative study. Clin Nutr ESPEN 2016;12:e14-9.

9. Doig GS, Simpson F; Early PN Trial Investigators Group. Early parenteral nutrition in critically ill patients with short-term relative contraindications to early enteral nutrition: a full economic analysis of a multicenter randomized controlled trial based on US costs. Clinicoecon Outcomes Res 2013;5:369-79.

10. De Jonghe B, Appere-De-Vechi C, Fournier M, Tran $\mathrm{B}$, Merrer J, Melchior JC, et al. A prospective survey of nutritional support practices in intensive care unit patients: what is prescribed? What is delivered? Crit Care Med 2001;29:8-12.

11. Dhaliwal R, Jurewitsch B, Harrietha D, Heyland DK. Combination enteral and parenteral nutrition in critically ill patients: harmful or beneficial? A systematic review of the evidence. Intensive Care Med 2004;30:1666-71. 